\title{
Successful Treatment of Fibrosing Organising Pneumonia Causing Respiratory Failure with Mycophenolic Acid
}

\author{
Christina Paula Ammy Lin-Shaw ${ }^{c}$ Mariamma Joseph ${ }^{b}$ Keith Kwan ${ }^{b}$ \\ Gianluigi Sergiacomi ${ }^{d}$ Marco Mura ${ }^{a}$ \\ ${ }^{a}$ Division of Respirology and ${ }^{\mathrm{b}}$ Department of Pathology, Western University, and ${ }^{\mathrm{C}}$ Department of Pharmacy \\ Services, London Health Sciences Centre, London, Ont., Canada; ${ }^{\mathrm{d} D i a g n o s t i c a}$ per Immagini e Radiologia \\ Interventistica, University of Rome Tor Vergata, Rome, Italy
}

\section{Established Facts}

- Fibrosing variants of organising pneumonia (OP) can occasionally occur with an aggressive presentation causing respiratory failure.

- The treatment of severe OP causing respiratory failure is not standardised.

\section{Novel Insights}

- Three cases of biopsy-proven fibrosing OP presented with a similar radiographic pattern of peribronchial and subpleural consolidations, and a fibro-inflammatory interstitial component was seen on the surgical biopsies.

- Mycophenolic acid provided clinical, functional, and radiographic improvement in a case series of severe OP with incomplete response to corticosteroid treatment.

\section{Key Words}

Organising pneumonia $\cdot$ Respiratory failure .

Mycophenolic acid

\section{Abstract \\ Organising pneumonia (OP) is usually promptly responsive to corticosteroid treatment. We describe a series of 3 cases of severe, progressive, biopsy-proven fibrosing OP causing respiratory failure. All cases presented with peribronchial}

and subpleural consolidations, had a fibro-inflammatory infiltrative component in the alveolar septa, and only had a partial and unsatisfactory response to corticosteroids. However, they responded to mycophenolic acid (MPA) treatment with resolution of respiratory failure as well as clinical and functional improvement. MPA as an additional treatment option for aggressive forms of fibrosing OP and interstitial lung disease needs to be further explored.

(c) 2016 S. Karger AG, Base

\section{KARGER}

E-Mail karger@karger.com www.karger.com/res (c) 2016 S. Karger AG, Basel

$0025-7931 / 16 / 0924-0279 \$ 39.50 / 0$
Marco Mura, MD, PhD

London Health Sciences Centre, Victoria Hospital

800 Commissioners Road East, Room E6-203

London, ON N6A 5W9 (Canada)

E-Mail marco.mura@lhsc.on.ca 


\section{Introduction}

Organising pneumonia (OP) is characterised by alveolar injury, granulation tissue buds, deposition of fibroblasts, and loose connective tissue within alveolar spaces [1]. The term 'cryptogenic' is used when no aetiology is identified [1]. Cryptogenic OP (COP) usually presents with multiple, bilateral, often migrating airspace opacities, restrictive ventilator defect, and a reduced diffusing lung capacity for carbon monoxide (DLCO) [2]. The mainstay of treatment of COP is represented by corticosteroids, with generally rapid response, complete resolution of pulmonary infiltrates, and normalisation of lung function $[2,3]$. Severe and/or rapidly progressive presentations of OP, leading to respiratory failure, have been described [4], but no standardised treatment is known.

The use of mycophenolic acid (MPA) for OP has never been described. MPA is the active component of mycophenolate mofetil and is a potent, selective, noncompetitive, reversibleinhibitor of inosine- $5^{\prime}$-monophosphate dehydrogenase. The efficacy of MPA in the treatment of interstitial lung disease (ILD) secondary to connective tissue disease is increasingly being recognised [5].

We describe a case series of biopsy-proven fibrosing OP leading to severe respiratory failure, with only partial response to corticosteroids, but with significant improvement after treatment with MPA.

\section{Case Report}

Case 1

A previously healthy, 67-year-old man was admitted with hypoxaemic respiratory failure following a 3 -week history of worsening dyspnoea not responsive to antibiotics, as well as progressive respiratory failure requiring an $\mathrm{FiO}_{2}$ of $50 \%$. Chest X-ray (CXR) showed diffuse airspace opacities. A chest CT scan demonstrated patchy bilateral consolidations, with a predominantly subpleural, peribronchial distribution. Bronchoalveolar lavage (BAL) cultures were negative. Lung biopsies were obtained with video-assisted thoracoscopy (VATS) from each lobe of the right lung. These revealed a diffuse, uniform pattern of $\mathrm{OP}$, with fibroblastic plugs in multiple airspaces, and also fibroblastic incorporation into the alveolar walls. In some areas, inflammatory cells, lymphocytes and plasma cells, were seen in the alveolar septa, although the process mainly involved airspaces. There were no granulomas, hyaline membranes, or vasculitis. No specific aetiology was found. Autoimmune causes were ruled out by extensive serologic workup and clinical examination. There was no exposure to drugs potentially causing OP, and no previous history of radiotherapy. Methylprednisolone $10 \mathrm{mg} / \mathrm{kg}$ i.v. for 3 days and oral prednisone $1 \mathrm{mg} / \mathrm{kg}$ were given. After 2 weeks of treatment on high-dose oral steroid, the patient developed steroid-induced diabetes, and a slow taper of corticosteroid was initiated (by $5 \mathrm{mg}$ every 7 days, until reaching
$15 \mathrm{mg}$ ), but he still needed 5 litres/min of oxygen, with persistent opacities on CXR. Oral MPA at $720 \mathrm{mg}$ b.i.d. was started. At the time of discharge, his FVC was 1.4 litres, $39 \%$ of predicted. The patient came off oxygen within 4 weeks since starting MPA. Lung function improved remarkably, with an FVC of 2.5 litres (73\% of predicted) and a DLCO of 77\% by week 18; the CXR abnormalities had nearly resolved (fig. 1). Low-dose prednisone was eventually completely stopped at week 24 since presentation.

\section{Case 2}

A 56-year old female with a remote history of breast cancer, who had never received chemotherapy, and with an 8-month history of slowly worsening dyspnoea presented to the hospital after failing two courses of antibiotic therapy. She was requiring an $\mathrm{FiO}_{2}$ of $35 \%$, and large consolidations with a predominantly subpleural and peribronchial distribution, mostly in the left lower lobe, were seen on CXR and CT. BAL was negative for pathogens. Her respiratory status continued to deteriorate, requiring mechanical ventilation. Two lung biopsies were obtained from the left lower lobe via VATS. These showed an acute-to-subacute airspace process and chronic, temporally homogeneous interstitial fibro-inflammatory disease, with no honeycomb lung. In several areas, fibroblastic polyps were not only seen in the airspaces, but also incorporated in the alveolar septa. No specific cause was identified. Autoimmune bloodwork was negative, and there was neither exposure to drugs potentially causing OP nor radiotherapy. She was given methylprednisolone at $10 \mathrm{mg} / \mathrm{kg}$ for 5 days, followed by oral prednisone at $1 \mathrm{mg} / \mathrm{kg}(70 \mathrm{mg})$ for 3 more weeks, with a taper starting on week 5. Due to her ongoing need for supplemental oxygen ( 5 litres/min), incomplete resolution of radiographic infiltrates, and significant steroid-induced myopathy starting after 3 weeks on high-dose corticosteroids, MPA at $720 \mathrm{mg}$ b.i.d. was started. In the subsequent weeks, her functional status consistently improved. After 16 weeks of MPA treatment, her FVC went from 1.8 to 2.3 litres, and DLCO from 29 to $66 \%$. She was weaned from oxygen, and her CXR showed improvement of the opacities (fig. 1). Prednisone was lowered to $15 \mathrm{mg}$ by week 12 .

\section{Case 3}

A 42-year-old female was referred for evaluation of progressive hypoxaemia. Three weeks prior to the referral, she had completed her third and last cycle of chemotherapy with cyclophosphamide, docetaxel, and trastuzumab for breast cancer. After unsuccessful empiric treatment with antibiotics, CXR showed bilateral, ill-defined airspace opacities, more severe on the left. These were confirmed on a chest CT scan, with a predominantly subpleural and peribronchial distribution, and ground-glass opacities in the upper lobes. BAL was negative for pathogens. Her respiratory status continued to decline, requiring mechanical ventilation. Lung biopsies from each lobe on the left were obtained via VATS. These demonstrated the presence of a diffuse, acute-to-subacute airspace process, with fibroblastic polyps, loose collagen, and fibroblast proliferation in the alveolar spaces. Several alveolar walls were thickened by fibroblast proliferation, oedema, and type 2 pneumocyte hyperplasia. The OP pattern was considered secondary to the recent chemotherapy. She was started on $1 \mathrm{mg} / \mathrm{kg}$ of oral prednisone and was able to be weaned off the ventilator. Five weeks later, although improved, she continued to require an $\mathrm{FiO}_{2}$ of $35 \%$, with persistent diffuse airspace opacities on CXR, and developed significant side effects from the use of corticosteroid. MPA was initi- 

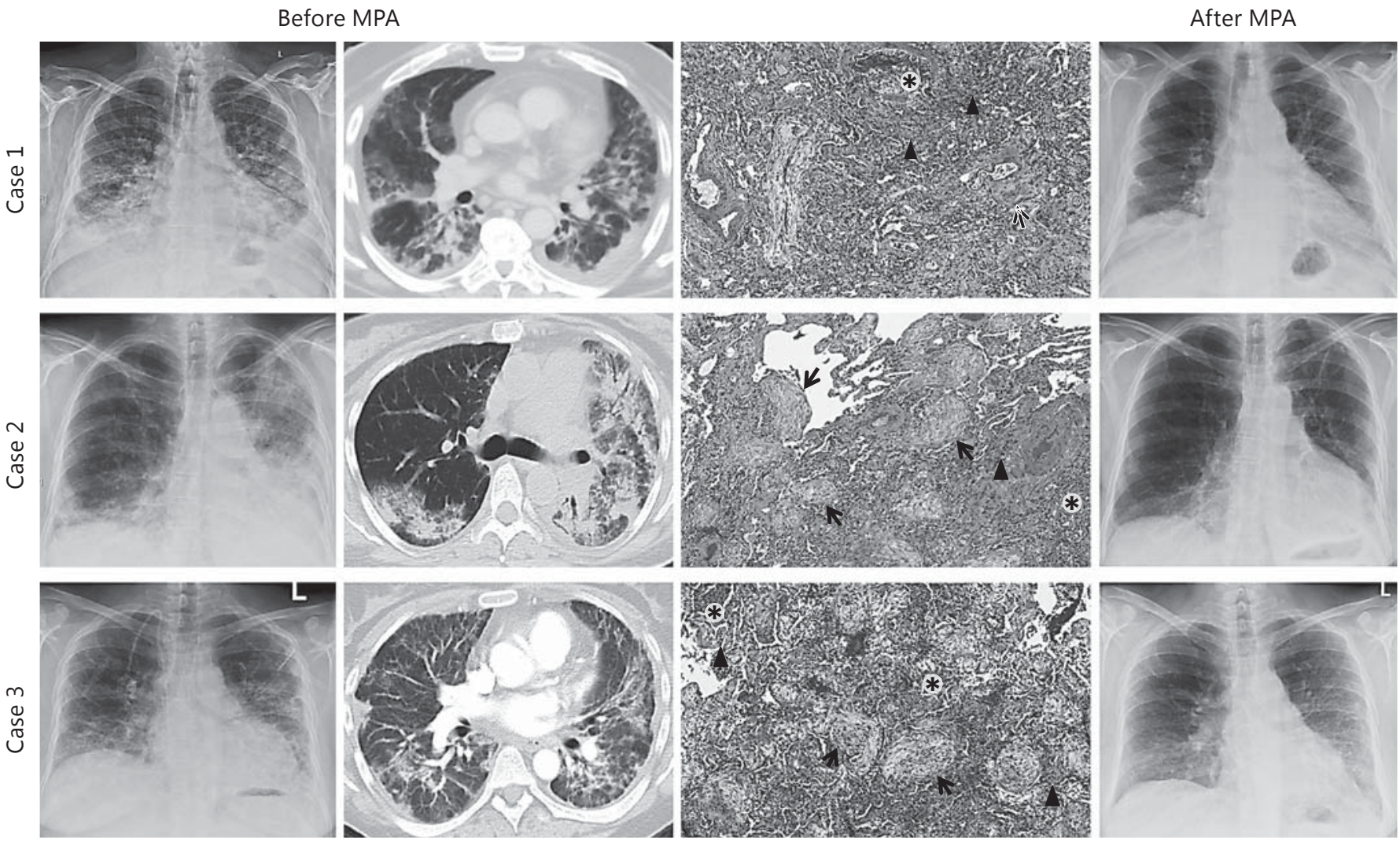

Fig. 1. From left to right: CXR and a chest CT scan before treatment with MPA, a representative pathology picture from surgical lung biopsy showing OP $(\times 10$ objective magnification), and CXR after treatment with MPA are shown for each of the 3 cases. In the pa-

thology panels, arrows indicate intra-alveolar fibroblastic polyps, arrowheads indicate areas of fibroblast and inflammatory cell interstitial infiltration, and asterisks indicate areas of intra-alveolar granulation tissue.

ated at the usual dose. Oxygen requirements soon improved, and she was completely weaned off oxygen in 14 weeks, while her exercise tolerance continued to improve. By week 25, her FVC went from 1.8 to 2.4 litres (72\%) and DLCO from 33 to $40 \%$. Prednisone was gradually reduced to $12.5 \mathrm{mg}$ daily by week 16 , and eventually stopped. The radiographic opacities improved (fig. 1).

\section{Discussion}

Cases of OP with an aggressive clinical course and development of respiratory failure are unusual but have been described [6-8]. While patients with COP do not usually develop respiratory failure, in the cases herein described hypoxaemia was severe and continued to worsen. Interestingly, in all cases the chest CT scans showed a pattern of extensive, patchy, bilateral consolidations, with a peribronchial and subpleural distribution. This pattern has been previously described in OP [2], but has not been

formally linked to a more aggressive clinical course. The lung biopsies showed extensive damage, with a prevalent, temporally homogeneous, and well-recognisable OP pattern, but also an interstitial component, with fibroblast proliferation and inflammatory cells causing alveolar wall thickening. No evidence of acute fibrinous OP, which usually has a severe presentation [9], was observed.

Rapid and complete recovery is the expected response to corticosteroids in OP [10]. A dose $\geq 40 \mathrm{mg}$ of prednisone was given, on average, for 7.5 weeks. Although our patients definitely improved to some degree with highdose corticosteroids, significant functional and radiographic abnormalities and the need for supplemental oxygen persisted. As we were faced with a lack of complete response and the development of significant adverse events from the use of high-dose corticosteroids, the choice of empiric MPA therapy was made, given the growing evidence on its efficacy in ILD $[5,11]$ and our own positive experience with the drug, which is mostly 
well tolerated $[5,11]$. MPA was effective in resolving respiratory failure in all 3 cases, with significant improvement of lung function and near resolution of radiographic opacities. Furthermore, corticosteroids were safely reduced.

Since MPA has never previously been described as a treatment option for OP, either cryptogenic or secondary to other causes such as chemotherapy, we can only speculate on its mechanism of action in this condition. The cytological profile of BAL in COP reveals a prevalence of lymphocytes, with a decreased CD4/CD8 ratio [12]. The activation of $\mathrm{T}$ cells is supported by the observed expression of human leukocyte antigen DR and, occasionally, interleukin-2 receptor [12]. MPA reduces the proliferation of T cells by arresting the synthesis phase of cell divi- sion [13]. It also suppresses the formation of adhesion molecules in endothelial cells, resulting in reduced recruitment of lymphocytes to sites of inflammation, with reduced production of cytokines [13].

In conclusion, MPA was remarkably effective as a second-line, supplemental therapy in this case series of unusually severe OP with only partial and unsatisfactory response to corticosteroids. This aggressive pathologic pattern, with a component of fibrosing interstitial involvement, was matched with the radiographic evidence of extensive peribronchial and subpleural consolidations. As the role of MPA in ILD is increasingly recognised, this report supports the conduction of larger cohort studies to ascertain benefits and risks of this additional therapy in OP.

\section{References}

$\checkmark 1$ Colby TV: Pathologic aspects of bronchiolitis obliterans organizing pneumonia. Chest 1992;102:S38-S43.

-2 Cordier JF, Loire R, Brune J: Idiopathic bronchiolitis obliterans organizing pneumonia. Definition of characteristic clinical profiles in a series of 16 patients. Chest 1989;96:9991004.

-3 Epler GR, Colby TV, McLoud TC, Carrington $\mathrm{CB}$, Gaensler EA: Bronchiolitis obliterans organizing pneumonia. N Engl J Med 1985;312: $152-158$.

4 Lee J, Cha SI, Park TI, Park JY, Jung TH, Kim $\mathrm{CH}$ : Adjunctive effects of cyclosporine and macrolide in rapidly progressive cryptogenic organizing pneumonia with no prompt response to steroid. Intern Med 2011;50:475479.

$\checkmark 5$ Fischer A, Brown K, Du Bois R, Frankel S, Cosgrove G, Fernandez-Perez E, Huie TJ, Krishnamoorthy M, Meehan RT, Olson AL, Solomon JJ, Swigris JJ: Mycophenolate mofetil improves lung function in connective tissue disease-associated interstitial lung disease. J Rheumatol 2013;40:640-646.

6 Nizami IY, Kissner DG, Visscher DW, Dubaybo BA: Idiopathic bronchiolitis obliterans with organizing pneumonia. An acute and life-threatening syndrome. Chest 1995; 108:271-277.

7 Yousem SA, Lohr RH, Colby TV: Idiopathic bronchiolitis obliterans organizing pneumonia/cryptogenic organizing pneumonia with unfavorable outcome: pathologic predictors. Mod Pathol 1997;10:864-871.

-8 Shitenberg D, Fruchter O, Fridel L, Kramer MR: Successful rituximab therapy in steroidresistant, cryptogenic organizing pneumonia: a case series. Respiration 2015;90:155159.

$>9$ López-Cuenca S, Morales-García S, MartínHita A, Frutos-Vivar F, Fernández-Segoviano P, Esteban A: Severe acute respiratory failure secondary to acute fibrinous and organizing pneumonia requiring mechanical ventilation: a case report and literature review. Respir Care 2012;57:1337-1341.

10 Barroso E, Hernandez L, Gil J, Garcia R, Aranda I, Romero S: Idiopathic organizing pneumonia: a relapsing disease. 19 years of experience in a hospital setting. Respiration 2007;74:624-631.

11 Swigris JJ, Olson AL, Fischer A, Lynch DA, Cosgrove GP, Frankel SK, Meehan RT, Brown KK: Mycophenolate mofetil is safe, well tolerated, and preserves lung function in patients with connective tissue disease-related interstitial lung disease. Chest 2006;130:30-36.

12 Costabel U, Teschler H, Guzman J: Bronchiolitis obliterans organizing pneumonia (BOOP): the cytological and immunocytological profile of bronchoalveolar lavage. Eur Respir J 1992;5:791-797.

13 Allison AC, Eugui EM: Mycophenolate mofetil and in mechanisms of action. Immunopharmacology 2000;47:85-118. 